\title{
Annihilation dynamics of topological defects induced by microparticles in nematic liquid crystals
}

DOI:

10.1039/C9SM01710K

Document Version

Accepted author manuscript

Link to publication record in Manchester Research Explorer

\section{Citation for published version (APA):}

Shen, Y., \& Dierking, I. (2019). Annihilation dynamics of topological defects induced by microparticles in nematic liquid crystals. Soft Matter. https://doi.org/10.1039/C9SM01710K

\section{Published in:}

Soft Matter

\section{Citing this paper}

Please note that where the full-text provided on Manchester Research Explorer is the Author Accepted Manuscript or Proof version this may differ from the final Published version. If citing, it is advised that you check and use the publisher's definitive version.

\section{General rights}

Copyright and moral rights for the publications made accessible in the Research Explorer are retained by the authors and/or other copyright owners and it is a condition of accessing publications that users recognise and abide by the legal requirements associated with these rights.

\section{Takedown policy}

If you believe that this document breaches copyright please refer to the University of Manchester's Takedown Procedures [http://man.ac.uk/04Y6Bo] or contact uml.scholarlycommunications@manchester.ac.uk providing relevant details, so we can investigate your claim.

\section{OPEN ACCESS}




\section{Soft}

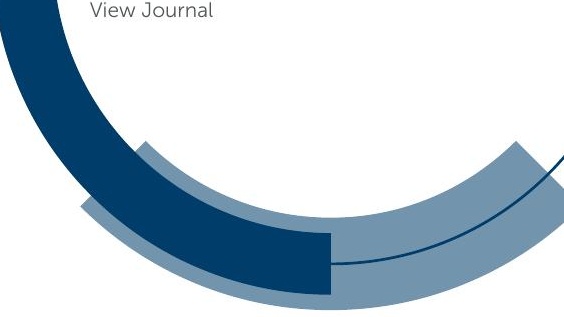

\section{Accepted Manuscript}

This article can be cited before page numbers have been issued, to do this please use: Y. Shen and I.

Dierking, Soft Matter, 2019, DOI: 10.1039/C9SM01710K.
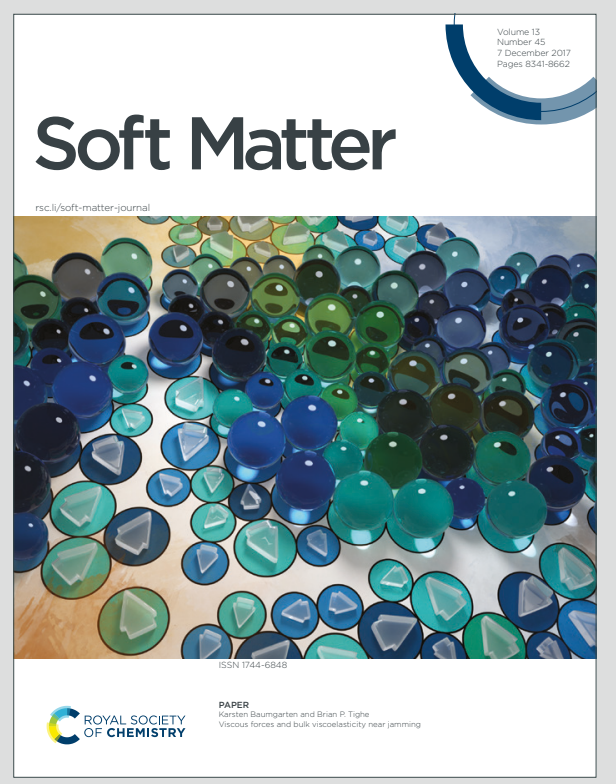

This is an Accepted Manuscript, which has been through the Royal Society of Chemistry peer review process and has been accepted for publication.

Accepted Manuscripts are published online shortly after acceptance, before technical editing, formatting and proof reading. Using this free service, authors can make their results available to the community, in citable form, before we publish the edited article. We will replace this Accepted Manuscript with the edited and formatted Advance Article as soon as it is available.

You can find more information about Accepted Manuscripts in the Information for Authors.

Please note that technical editing may introduce minor changes to the text and/or graphics, which may alter content. The journal's standard Terms \& Conditions and the Ethical guidelines still apply. In no event shall the Royal Society of Chemistry be held responsible for any errors or omissions in this Accepted Manuscript or any consequences arising from the use of any information it contains. 


\section{ARTICLE}

\section{Annihilation dynamics of topological defects induced by microparticles in nematic liquid crystals}

Received 00th January 20xx, Accepted 00th January 20xx

DOI: $10.1039 / x 0 x x 00000 x$

\begin{abstract}
Yuan Shen, ${ }^{a}$ Ingo Dierking *a
The annihilation dynamics of $s= \pm 1$ topological defects with and without microparticles in a nematic liquid crystal are investigated and compared. The microparticle with homeotropic surface anchoring can act as a $s=$ +1 defect and produce a corresponding $s=-1$ defect nearby. Both of them attract and move towards each other. The speed of the positive defect induced by microparticle is much slower than that of the negative defect, contrary to the situation without particle. The effects of electric field strength and frequency, particle size, confining cell gap, temperature were systematically investigated. The study shows that the dynamics of the annihilation process is related to a complex interplay between elastic attractions, viscous drag forces, backflow effects, director configurations and cell confinement.
\end{abstract}

\section{Introduction}

Topological defects have been observed in various areas of physics from cosmic strings ${ }^{1}$ to vortices in superfluid helium ${ }^{2}$. The study of the dynamics of those topological defects is an increasingly central problem in particle physics, condensed material physics, and cosmology ${ }^{3}$. However, the investigation of topological defect dynamics in many condensed materials is restricted by the motionless background such as flux line dynamics in superconductors ${ }^{4}$. Fortunately, liquid crystals (LCs), in which the coupling of the propagation of defects and flows is expected, provide an ideal system for studying the dynamics of topological defects due to the variety of defectrelated phenomena and relatively easy observation and manipulation caused by the very small elastic constants in comparison to solid state materials ${ }^{5}$.

In a system where nematic LCs are confined by continuously degenerate boundary condition, topological defects with four essential molecular arrangements, $s= \pm 1$ and $s= \pm 1 / 2(s$, topological strength), can be detected, which is well known as the Schlieren texture ${ }^{6}$. Between crossed polarizers, these defects are connected via a network of two- or fourfold black brushes, showing the regions where the optical axis is parallel to one of the polarizers ${ }^{7}$. To maintain the conservation of the total topological charge, the sum of the overall topological strength of defects is zero. This strictly holds for an infinitely large sample, but is also approximated very well in real systems with liquid crystals. Due to the elastic deformation of the director field,

\footnotetext{
a. School of Physics and Astronomy, University of Manchester, Oxford Road, Manchester M13 9PL, UK.Email: ingo.dierking@manchester.ac.uk

+ Footnotes relating to the title and/or authors should appear here.

Electronic Supplementary Information (ESI) available: [details of any supplementary information available should be included here]. See DOI: 10.1039/x0xx00000x
}

defects with equal topological strength but opposite sign attract each other and annihilate to minimize the free energy of the system, eventually resulting in a uniform director configuration.

The formation of topological defects in LCs is generally realized by a rapid quench of temperature or pressure across the isotropic to LC phase transition. Such a sudden symmetrybreaking phase transition produces a dense tangle of topological defects and is well described by the Kibble-Zurek mechanism ${ }^{8}$, 9. However, in this investigation, the defects are created by a different mechanism. A nematic LC with negative dielectric anisotropy is subjected to homeotropic boundary conditions. Application of an electric field normal to the cell substrates with a value above the Frederiks threshold, causes a reorientation of the director into planar condition, while umbilic defects are formed due to the lack of a preferred direction of the planar state 7,10 . These umbilic defects are favourable for the investigation of defect dynamics because their configurations are very well defined with fixed topological strength of $s= \pm 1$ only ${ }^{11,12}$.

The investigation of the annihilation dynamics of topological defects has been the focus of both theoretical simulations ${ }^{13-16}$ and experimental studies 11, 12, 17-23. The first experiment concerning defect annihilation was probably reported by Williams et al. who observed point hedgehog defects in cylindrical capillaries filled with nematic LCs ${ }^{24}$. After that, studies not only related to point defects ${ }^{25-28}$, but also to string defects $^{3,29,30}$, defect loops ${ }^{31}$ and umbilic defects ${ }^{11,12}$ have been reported. In most of those studies, an anisotropy in the speed at which defects of opposite strength approach each other was observed. Surprisingly, all of these studies consistently demonstrated that positive defects move faster than negative ones, which was attributed to their different hydrodynamic properties ${ }^{13,14}$.

Topological defects are also of great interest from the standpoint of trapping and manipulation of colloidal inclusions 32-35. Dispersions of microparticles in nematic LCs have been 
investigated since topological defects of LCs were studied in more detail. It has been demonstrated that these microparticles will break the symmetry configuration of the nematic phase and thus producing defects ${ }^{5,36-39}$. So far, a considerable number of studies related to LC colloids ${ }^{40-45}$ have been reported which greatly enrich our knowledge about their physical properties. However, as far as we are aware, the investigations on the annihilation dynamics of defects in LC colloids have rarely been explored. This aspect is important for a general understanding not only of the interactions between particles and defects ${ }^{46-48}$, but also their dynamics.

Here, we demonstrate a dynamic process where a colloidal microparticle acts as a positive defect, and attracts a negative defect nearby. As a result, both of them move towards each other and leading to a motion similar to the annihilation process of two defects with opposite signs.

\section{Materials and methods}

A commercially available LC mixture, ZLI-2806 from Merck was used for the investigations. It has a negative dielectric anisotropy of $\Delta \varepsilon=-4.8$ and a phase sequence on cooling of Iso $\left(100{ }^{\circ} \mathrm{C}\right) \mathrm{N}\left(-20^{\circ} \mathrm{C}\right)$ Cryst. ${ }^{9}$. The dispersed microparticles are silica beads with diameters of $6 \mu \mathrm{m}, 10 \mu \mathrm{m}, 15 \mu \mathrm{m}$ and $23 \mu \mathrm{m}$, respectively. The size distribution of the particles can be found in Fig. S1. These silica microparticles were treated with Dimethyloctadecyl [3-(trimethoxysilyl) propyl] ammonium chloride (DMOAP) to induce homeotropic surface anchoring. Standard sandwich cells with cell gaps of $11 \mu \mathrm{m}, 21 \mu \mathrm{m}, 32 \mu \mathrm{m}$ and $43 \mu \mathrm{m}$ were house made of ITO coated glass. The cells had a well-defined thicknesses $( \pm 0.5 \mu \mathrm{m})$. The ITO glass used was first washed with methanol and isopropanol and then coated with DMOAP to exhibit homeotropic alignment.

A very small amount of microparticles was placed at the entrance of the individual cells in order to be dragged in by capillary filling of the LC. The concentration of the particles is not quantified. However, in most cases, the regions we choose to conduct our investigations have less than 5 particles with an area as large as $770 * 409 \mu^{2}$. It has been reported that microparticles dispersed in LC matrixes will be repelled from the cell substrate by elastic forces due to the incompatibility of the uniform director field near the substrate with the director distortions around the particles ${ }^{49-51}$. Conflicting reports, which claim that the particles may get stuck onto the cell substrate ${ }^{32}$ can also be found. To assure the particles were located in the bulk, we applied short electric pulses to induce hydrodynamic flow to push particles into LC bulk ${ }^{32}$. Measurements were started well after the hydrodynamic flow died down and the induced distortions had relaxed.

Samples were observed in a polarized optical microscope (POM, Leica OPTIPOL) with the motion of particles and defects recorded via a digital camera (UI-3360CP-C-HQ, uEye Gigabit Ethernet) at a resolution of $2048 * 1088$ pixel. The recorded videos were then analysed via an open source software ImageJ. Sinusoidal wave alternating electric fields were applied by a function generator (33220A, Agilent) in combination with a home-made power amplifier.

\section{Results and discussion}

View Article Online DOI: 10.1039/C9SM01710K Fig. 1 (a) shows a texture of the micro-particle in a LC sample observed between crossed polarizers, additionally providing the corresponding LC director configuration. The LC director field far away from the particle is determined by the homeotropic boundary conditions of the substrates. The LC director near the particle is distorted due to the homeotropic surface anchoring on the particle, producing a line defect encircling the particle at the equator, i.e. the Saturn ring defect. An electric field perpendicular to the substrates is then applied to the sample. Due to the negative dielectric anisotropy, the LC molecules realign themselves perpendicularly to the electric field direction, resulting in a symmetry-breaking transition which is accompanied by the formation of a huge amount of umbilic defects. To minimize the free energy, the particle itself acts as a radial hedgehog defect $(s=+1)$ which is connected with several hyperbolic hedgehog defects $(s=-1)$ as in Fig. 1 (b). According to the investigation by Fowler et al., the formation of defects is sensitive to the ramp rate of the applied electric field, the higher the ramp rate, the larger the defect formation density ${ }^{9}$. In our experiment such a phenomenon is also confirmed. When a low electric field is applied to the sample, e.g. $0.3 \mathrm{~V} / \mu \mathrm{m}$, the particle $(s=+1)$ is usually connected with only one negative defect ( $s=$ -1) through all the four black brushes as depicted in Fig. 1 (b). But when a high electric field is applied on the sample, e.g. 1.0 $\mathrm{V} / \mu \mathrm{m}$, there will usually be two or more negative defects $(s=-$ 1) connecting with the particle through only one or two black brushes. According to our investigation, the number of the black brushes, $N$, connected with the particle and the defects will severely influence the annihilation velocities of particles and defects, which will be discussed below. In order to maintain consistency throughout the experiments, the samples were first subjected to a low electric field, which was then gradually increased to higher values. This process guarantees that the defect-particle couples investigated are always connected with each other through all the four black brushes and isolated from other defects or particles. Due to the elastic attraction force, $F_{\mathrm{a}}$, induced by distorted regions around the particle and the defect ${ }^{23}$, they slowly move towards (Fig. S2, Supplementary Movie 1) and finally combine with each other, leading to a motion similar to the annihilation of two defects with opposite signs. As a result, the initial Saturn ring defect encircling the particle in Fig. 1 (a) transforms into a hyperbolic hedgehog defect located close to the particle in order to satisfy topological constraints, Fig. 1 (c). It should be noted that the transition from Fig. 1 (a) to (c) is dependent on the thickness of the sample $d$, as well as the diameter of the particle $R$. If $d / R$ is too large (here $>3.5$, with 6 $\mu \mathrm{m}$ particles filled in $21 \mu \mathrm{m}$ thick cells, where the particles cannot induce the negative hyperbolic defect), the particle will induce the hedgehog defect in Fig. 1 (c) directly, when an electric field is applied. The annihilation process of Fig. 1 (b) will not happen. It should also be noted that the homeotropic anchoring of the particle surface is necessary for the occurrence of the annihilation process. If the anchoring of the particle surface is homogenous, the particles will induce boojum defects, Fig. S3. 
Fig. 1 (d) depicts a typical trajectory during the annihilation process of the particle $(s=+1)$ and its counterpart defect $(s=$ 1). From the figure we can observe the typical "S-shape" trajectory which is generally observed in the annihilation process of two opposite defects with no particles ${ }^{52-54}$. In addition, at this point it can already clearly be observed that the negative defect is moving faster than the particle. Fig. 1e shows the director field at the early stage of the annihilation, where the director field

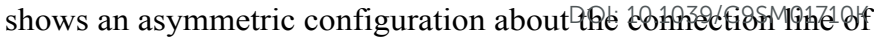
the particle and the defect centres. Fig. If shows a symmetric configuration at the late stage of the annihilation. The transformation of the director field from (e) to (f) leads to the typical "S-shape" trajectory of the annihilation of defects.
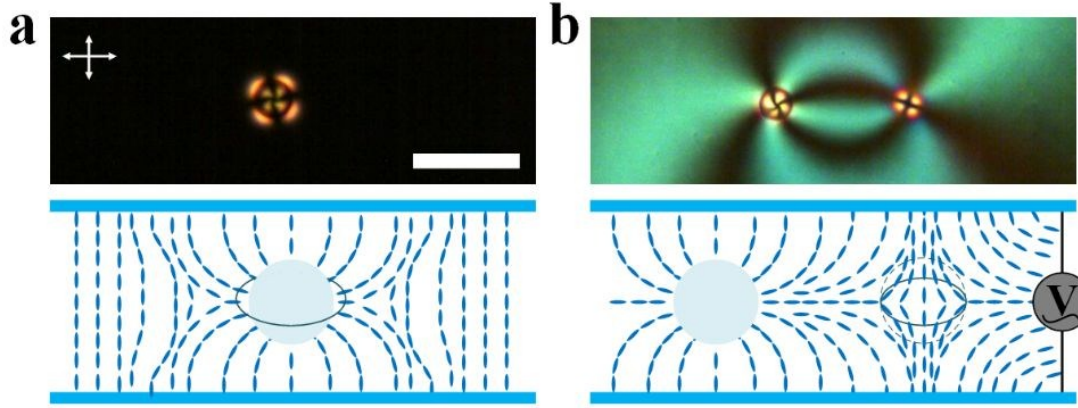

d

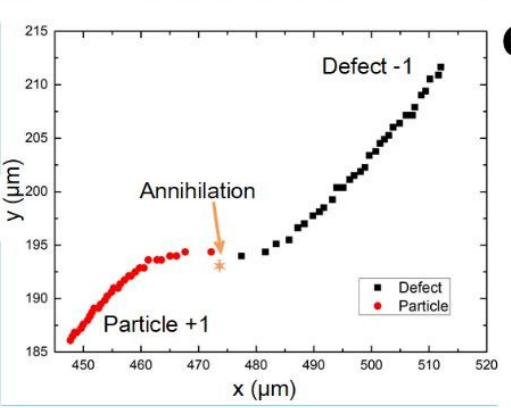

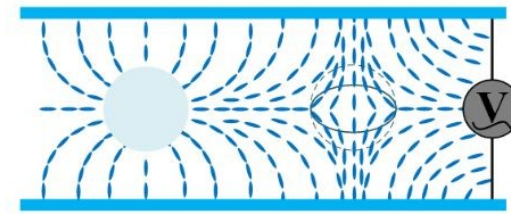
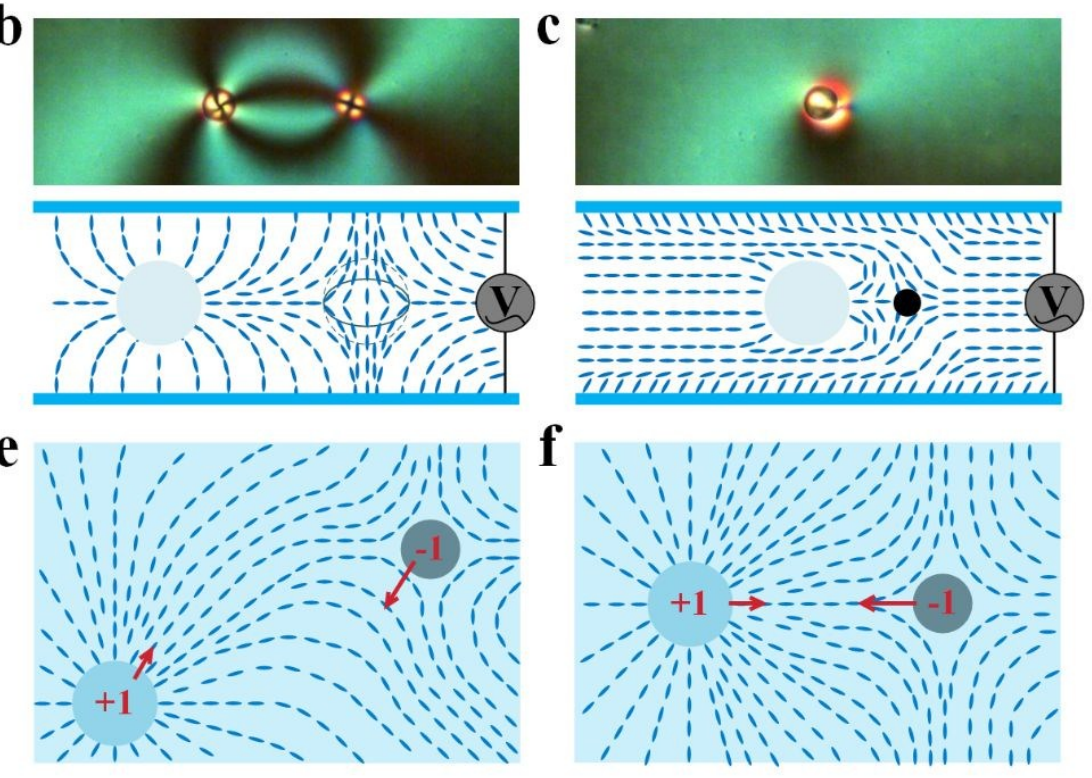

Fig. 1. The POM image (upper) of the particle $(R=15 \mu \mathrm{m})$ and the negative defect in a cell $(d=21 \mu \mathrm{m})$ with homeotropic alignment, as well as the corresponding sketch of the LC director distribution (bottom). (a) the Saturn ring defect encircles the particle without applying electric field. The scale bar is $50 \mu \mathrm{m}$. (b) the radial hedgehog defect (particle, $s=+1)$ and the corresponding hyperbolic hedgehog defect $(s=-1)$ at electric field $E=0.3 \mathrm{~V} / \mu \mathrm{m}, f=1 \mathrm{KHz}$. (c) the hedgehog defect of the particle after the annihilation of (b) at $E=0.3 \mathrm{~V} / \mu \mathrm{m}, f=1 \mathrm{KHz}$. (d) Exemplary trajectories of a particle $(R=10 \mu \mathrm{m}, \mathrm{s}=+1$, red circles) and the corresponding hyperbolic hedgehog defect ( $\mathrm{s}=-1$, black squares) during the annihilation process at a time resolution of 1 second. The orange star represents the point of the annihilation. The electric field is $E=0.75$ $\mathrm{V} / \mu \mathrm{m}, f=1 \mathrm{KHz}$, cell gap $d=21 \mu \mathrm{m}$, temperature $T=26^{\circ} \mathrm{C}$. (e) and (f) are the corresponding 2D director configurations of the particle and the defect at the early and late stages of annihilation, respectively (top view). The red arrows represent the velocities.

Fig. 2 (a) depicts the distances travelled by two topologically opposite defects without particles during the whole annihilation process at varied amplitudes of electric fields. According to previous studies ${ }^{11,25,55,56}$, the separation $D$ between two defects with opposite topological strength is expected to scale with time as

$$
D \propto\left(t_{0}-t\right)^{\alpha}
$$

Where $t_{0}-t$ is the time to annihilation, $\alpha$ is the annihilation separation exponent. The elastic attraction force, $F_{\mathrm{a}}$, between two defects can be expressed as 57,58

$$
F_{a}=2 \pi s_{1} s_{2} K / D
$$

where $s_{1}, s_{2}$ are the topological strength of defects, $K$ is the elastic constant. At early times, long before annihilation, where $D$ is much larger than the cell gap $d$ (usually, $>2 d$ ), $F_{\mathrm{a}}$ can be nearly regarded as a constant, and the distance travelled is directly proportional to time, i.e. $\alpha=1.0$. On the contrary, at the late stages, closer to the annihilation, this constant attraction force transforms into a separation-dependent force, and the square root behaviour, i.e. $\alpha=$
0.5 , is observed. As shown in Fig. 2(a), at the early stage of the annihilation process, the distance travelled is directly proportional to time, for both defects. Thus, it is justified to estimate defect speeds at the early stages of annihilation via a linear distance-time relationship ${ }^{11}$. This generally linear behaviour is also observed for the motion of the particle-defect couple as shown in Fig. 2 (b). As a result, the velocities of both defects and particles discussed below are determined by the slope of linear distance-time relationship at the early stage of annihilation $(D \geq 2 d)$. In addition, in Fig. 2 (a), it is clearly observed that, without particles, the distance travelled by the $s=+1$ defect is larger than the one travelled by the $s=-1$ defect within the same duration. This behaviour implies that the positive defect moves faster than the negative one ${ }^{14,15}$.

However, this is not the case with the particle-defect couple, where the distance travelled by the positive defect (particle), is much smaller than the one travelled by the negative defect, Fig. 2 (b). This behaviour demonstrates that the positive defect (or the particle) 
moves much more slowly than the negative defect. At high electric fields $(E>1.0 \mathrm{~V} / \mu \mathrm{m})$ this behaviour can be pushed to a situation where the particle does not move at all. It should be noted that at the late stage of the annihilation, when the negative defect gets very close to the particle, the radial hedgehog defect core $(s=+1)$ at the centre of the particle will be attracted by the negative defect and extracted from the particle. At this moment, the defect core is separated from the particle centre and then combines with the negative defect.
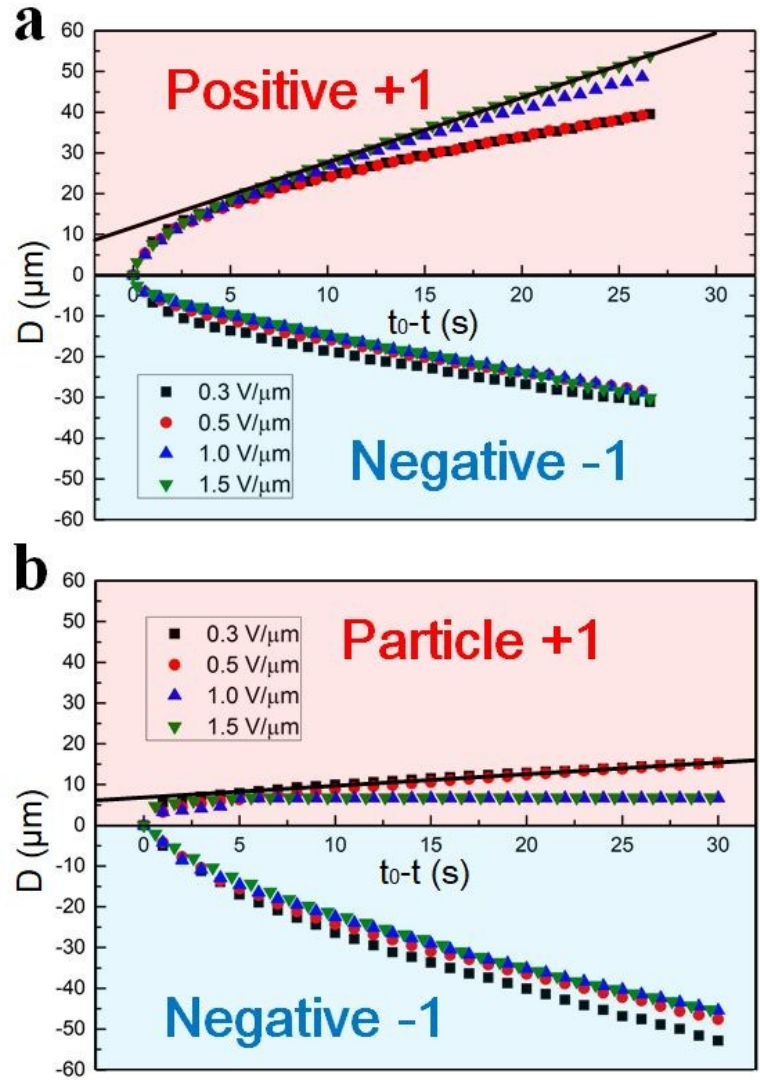

Fig. 2. The relationships between the annihilation time and the travelled distance of (a) positive and negative umbilic defects and (b) the particle $(s=+1)$ and the corresponding hyperbolic hedgehog defect $(s=-1)$. During the early times of the annihilation process, the distances that the defects and the particles travelled are approximately linearly proportional to time, thus allowing an easy determination of the speeds of defects and particles. The frequency of the electric field $f=1 \mathrm{KHz}$, particle size $R=15 \mu \mathrm{m}$, cell gap $d=21 \mu \mathrm{m}$, the number of brushes $N=4$, room temperature $(T$ $=26^{\circ} \mathrm{C}$.

Furthermore, it is also observed that the motion of the defects, especially the positive ones in Fig. 2 (a), is dependent on the amplitude of the electric field. This dependence is carefully investigated in Fig. 3 (a) where we can establish that firstly, at low electric fields $(<0.6 \mathrm{~V} / \mu \mathrm{m})$, the velocity of the positive defect $(s=$ +1 ) increases gradually with the increase of the electric field. On further increase of the electric field, the velocity drastically increases and finally saturates at high electric fields $(>1.5 \mathrm{~V} / \mu \mathrm{m})$. However, the velocity of the negative defect $(s=-1)$ firstly decreases and then gradually increases before it also saturate@/atohigh/fresdls?.1Thke increase of the velocity of both defects can be attributed to backflow effects. According to the investigation by Oswald et al ${ }^{20}$, at low voltage, the LC molecules align homeotropically over a large surface area inside the core of the defects, which will efficiently inhibit the backflow effects. With increasing the voltage, more molecules tend to homogeneously align in the bulk, leading to larger backflow. However, when the voltage is high enough, the backflow effect will be localized close to the cores of the two defects and does not vary with the voltage any longer. In other words, at low electric fields, the backflow effect grows gradually and shows a parabolic dependence on the electric field ${ }^{15}$. While at high electric fields, the backflow effect is saturated and does not change with electric field considerably. This dependence of backflow on electric field can further be proved by the increase of the velocity difference between two defects, $\Delta v$, with increasing electric field, as shown in the inset of Fig. 3 (a), since larger backflow effects will result in larger $\Delta v^{13}$, 14,18
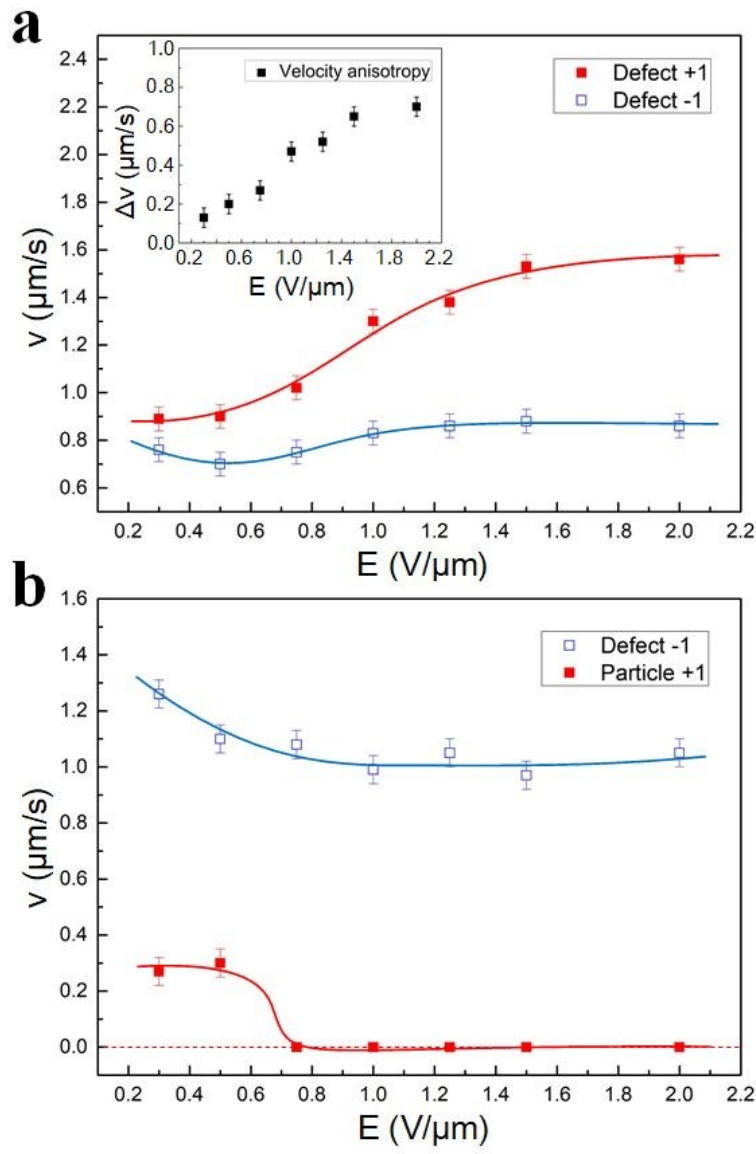

Fig. 3. The velocities of positive defects $(s=+1$, red squares) without (a) and with particles (b) as well as negative defects ( $s=-1$, blue squares) as a function of the amplitude of electric field. The frequency of the electric field $f=1 \mathrm{KHz}$, particle size $R=15 \mu \mathrm{m}$, cell gap $d=21 \mu \mathrm{m}$, the number of brushes $N=4$, room temperature ( $T$ $=26^{\circ} \mathrm{C}$ ). The inset in (a) shows the dependence of velocity difference, $\Delta v$, on the electric field amplitude. $\Delta v$ is calculated by subtracting the velocity of positive defect with the velocity of the 
corresponding negative defect. The error bars are estimated from variation in velocities of several repeat experiments.

Besides, it can also be found that the overall increase of the velocity of the negative defect is much smaller than the positive one, which is due to the fact that the backflow around the negative defect is much weaker than the one around the positive defect. It has been reported that there are two sources of backflow ${ }^{14,21}$. The first one is the defect core. Because the nematic order is suppressed in the core and the viscosity at the core is usually larger. The motion of the core will induce vortices which are similar to those produced by a solid cylinder moving through a fluid. This flow is independent on the sign of the defect. The second source is the reorientation of the director field outside the core, which is dependent on the sign of the defect. It was revealed by numerical simulations ${ }^{13,14}$ that these two sources of flow reinforce each other for the positive defect, but partially cancel for the negative one, thus giving rise to the asymmetric annihilation of the defects.

It should be noted that there is a decrease of the velocity of the negative defect at low electric fields $(E=0.5 \mathrm{~V} / \mu \mathrm{m})$, which we think should be attributed to the increase of the frictional force. The frictional force of defects, $F_{\mu}$, can be expressed as ${ }^{59,60}$

$$
F_{\mu}=\mu \ln (D / r) v
$$

where $\mu$ is the (inverse) mobility of the defect, $r$ is the size of the defect core, and $v$ is the velocity of the defect. The size $r$ of the umbilic defect is dependent on the electric field, which can be determined by ${ }^{10}$ :

$$
r=\left(\frac{d}{\pi}\right) \cdot\left(\frac{E_{c}^{2}}{E^{2}-E_{\mathrm{c}}^{2}}\right)^{\frac{1}{2}}
$$

where $E_{\mathrm{c}}$ is the Freedericksz threshold electric field, above which the LC molecules in the mid-plane of the slab tend to tilt; $d$ is the thickness of the LC sample. As can be seen, $r$ continuously decreases as the amplitude of electric fields increases (in our case $r$ decreases from $\sim 16$ to $\sim 7 \mu \mathrm{m}$ as $E$ increases from 0.3 to $0.5 \mathrm{~V} / \mu \mathrm{m}$ in the experiment), leading to an increase of $F_{\mu}$. At high electric fields, $r$ does no longer change significantly. The reason why the positive defect does not show such a decrease should be attributed to the compensation from the larger backflow effect ${ }^{13,21}$.

Fig. 3 (b) shows the dependence of the velocities of the particles and the negative defects on the amplitude of the electric field. The velocity of the negative defects first slightly decreases at low electric fields, which is similar to the results in Fig. 3 (a). On the other hand, the velocity of the positive defects (particles), is drastically decreased, which is not only much slower than the positive ones in Fig. 3 (a), but also slower than the negative defects. This abnormal deceleration, as we mentioned before, can be attributed to the strong viscous drag force of the particles. Additionally, the velocity of the particles also shows an independence on the electric fields at low amplitudes. Interestingly, when the electric field is increased even higher, the velocity of the particles suddenly decreases to zero. Subsequent reduction of the electric field causes some particles to move again. This behaviour of non-moving particles may be attributed to the pinning effect ${ }^{20}$ or the Freedericksz-like positional transition of particles reported recently that pushes particles towards the substrates ${ }^{61}$.
Fig. 4 (a) and (b) show the dependence of the defect annihilation separation scaling exponent $\alpha$ on the ampliญdeodp edecticl fields; with the inset in Fig. 4 (a) providing an example of typical experimental data used for the calculation of the exponent $\alpha$. The results in Fig. 4 (a) and (b) show that the exponents $\alpha$ for both defectdefect couples and particle-defect couples are independent on the applied electric field conditions, in accordance with the expected value of $\alpha \sim 0.5$ from theory and experiments ${ }^{12}$. It is interesting to note that the annihilation exponents of the particle-defect couples are also in the range of 0.5 , despite the fact that the particles do not move at all at high electric fields. This warrants further investigations in the future.

To investigate the influence of the cell gap on the annihilation dynamics of particle-defect couples, particles with equal diameter are dispersed in cells at varied cell gaps. Fig 5 depicts the dependences of the velocities of particles and defects as a function of electric field amplitude in cells with thicknesses $21 \mu \mathrm{m}$ (blue circles), $32 \mu \mathrm{m}$ (black squares) and $43 \mu \mathrm{m}$ (red trangles). The velocities of negative defects decrease with increasing cell gaps. This effect may be attributed to the larger initial separation distance $D_{i n}$, the separation distance $D$ between the particle and the negative defect at the time when the electric field is just applied, as shown in Fig. S2.
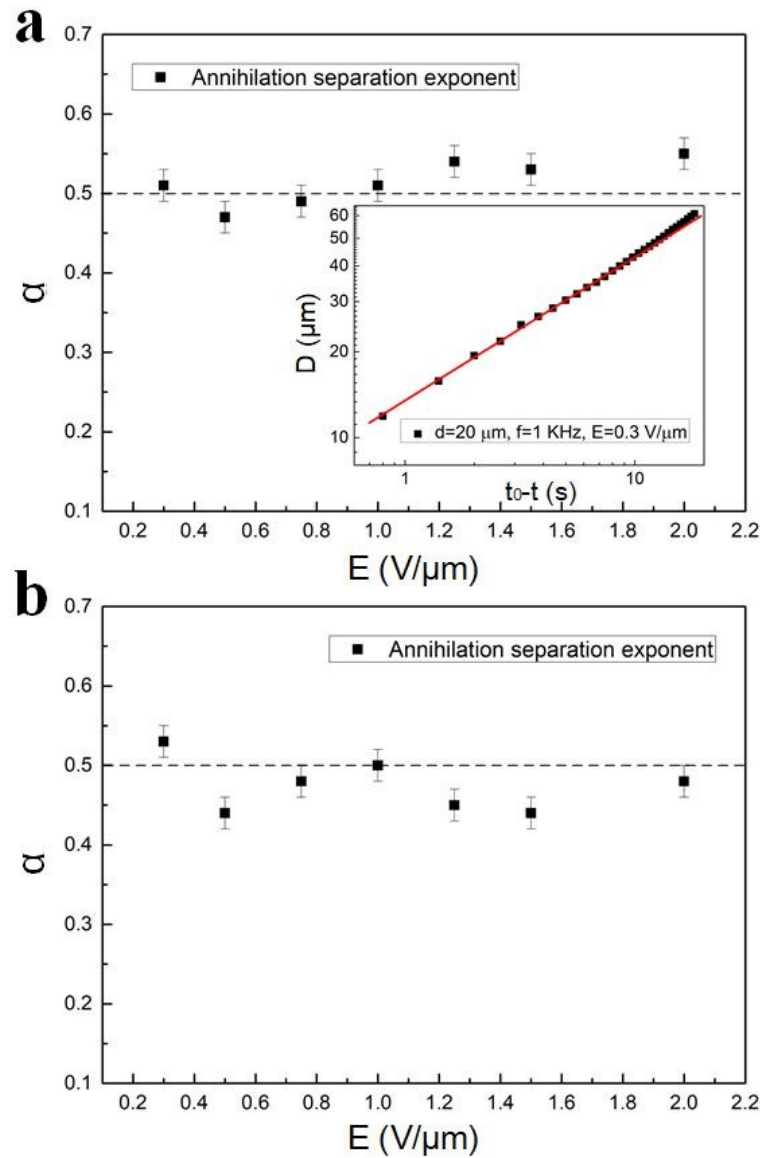

Fig. 4. The annihilation separation exponents of (a) the positivenegative defect couple and (b) the particle-negative defect couple as a function of the applied electric field amplitude. The inset in (a) is the exemplary experimental data for the determination of the defect pair annihilation separation exponent $\alpha$ for umbilical defects 
induced at electric field $E=0,3 \mathrm{~V} / \mathrm{um}$. The frequency of the electric field $f=1 \mathrm{KHz}$, particle size $R=15 \mu \mathrm{m}$, cell gap $d=21 \mu \mathrm{m}$, the number of brushes $N=4$, room temperature $\left(T=26^{\circ} \mathrm{C}\right)$. The error bars are estimated from variation in annihilation separation exponents of several repeat experiments.

This distance is larger for thicker cells, which effectively reduces $F_{\text {a }}$ between defect-particle couples. It shall here be demonstrated that $D_{\text {in }}$ is dependent on the cell gap as well as the diameter of particles. Both increase of cell gap, $d$, as well as diameter of particles, $R$, will lead to larger $D_{i n}$ between particles and negative defects. The specific dependence of $D_{i n}$ on the cell gap $d$ as well as on the particle diameter $R$ can be found in table $\mathrm{S} 1$. On the other hand, despite the fact that particle velocities in all cells are independent on electric field before suddenly becoming zero at high amplitudes, they increase with increasing cell gaps, which may be due to the weaker surface confinement in thicker cells. At low electric fields, it was also found that the velocities of negative defects in all cells exhibit a decrease, which can be attributed to the increase of $F_{\mu}$ as mentioned before. On the other hand, at high field amplitudes, we find that there is an increase of the negative defect velocity with increasing electric fields, and this increase becomes larger with increasing cell gaps. This can be attributed to the larger backflow effects in thicker cells ${ }^{62}$. As the boundary anchoring effect on LC molecules is large in thin cells, which suppress backflow effects. By increasing cell gap, the surface-imposed anchoring becomes weaker ${ }^{11}$. At the same time, the increasingly larger regions around the defect core involved in director reorientation results in stronger vortices and hence increases the backflow effects, too ${ }^{15}$.

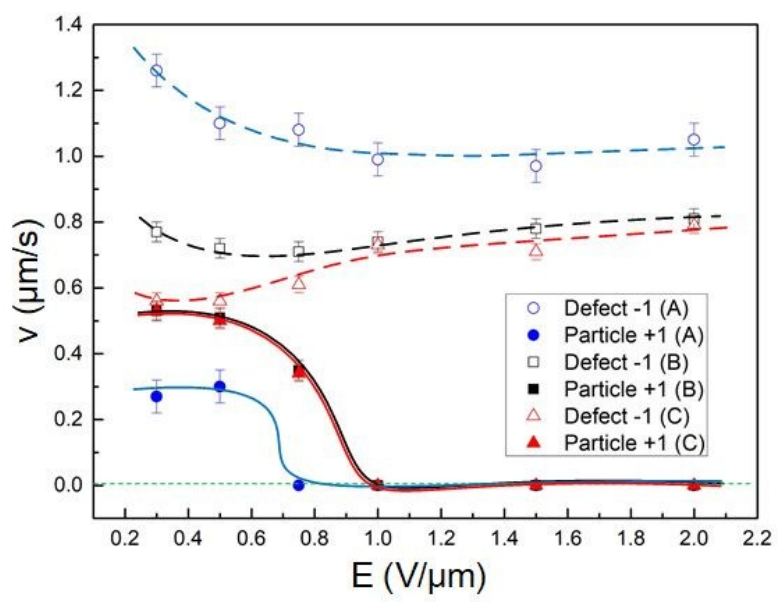

Fig. 5. The dependence of the velocities of negative defects (hollow symbols) and particles (solid symbols) on the amplitude of electric field. (A) $R=15 \mu \mathrm{m}, d=21 \mu \mathrm{m}$. (B) $R=15 \mu \mathrm{m}, d=32 \mu \mathrm{m}$. (C) $R=15$ $\mu \mathrm{m}, d=43 \mu \mathrm{m}$. The frequency of the electric field $f=1 \mathrm{KHz}$, the number of brushes $N=4$, room temperature $\left(T=26^{\circ} \mathrm{C}\right)$. The error bars are estimated from variation in velocities of several repeat experiments.

Furthermore, we investigated the influence of particle size on the annihilation process by investigating particles with different diameters in cells with equal gaps. Fig. 6 depicts the dependences of

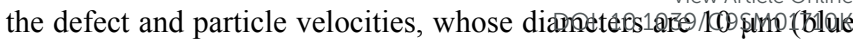
circles), $15 \mu \mathrm{m}$ (black squares) and $23 \mu \mathrm{m}$ (red trangles), on the amplitude of electric fields for cells with fixed gap $d=32 \mu \mathrm{m}$. It is found that the velocity of particles becomes larger by decreasing the particle size. The reason why the $23 \mu \mathrm{m}$ particles do not move may be that the elastic attraction is not strong enough to overcome the viscous drag. Another explanation may be that they are heavy enough to be stuck on the bottom cell substrate immediately after the electro-hydrodynamic effect ceases. On the other hand, the relationship between the negative defect's velocity and particle size seems not to be intuitive. At low electric fields, the negative defects, corresponding to the $10 \mu \mathrm{m}$ particles, move fastest, but by increasing the electric field, they strongly slow down and become slower than that of the negative defects corresponding to the $15 \mu \mathrm{m}$ particles. This complex transition may be attributed to the subtle balance between $F_{\mathrm{a}}$ and $F_{\mu}$. On the other hand, the negative defects corresponding to the $23 \mu \mathrm{m}$ particles, move most slowly which is due to the relatively small $F_{a}$ induced by the larger $D_{i n}$.

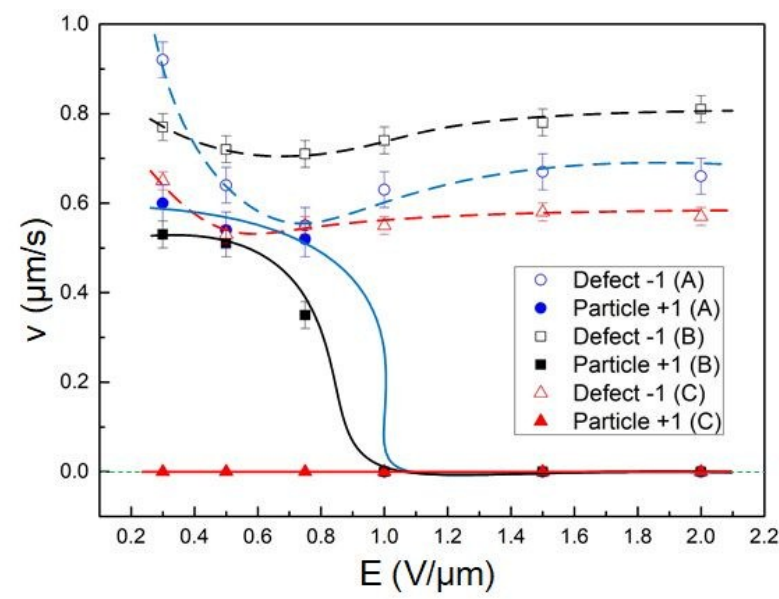

Fig. 6. The dependence of the velocities of negative defects (hollow symbols) and particles (solid symbols) on the amplitude of electric field. (A) $R=10 \mu \mathrm{m}, d=32 \mu \mathrm{m}$. (B) $R=15 \mu \mathrm{m}, d=32 \mu \mathrm{m}$. (C) $R=23$ $\mu \mathrm{m}, d=32 \mu \mathrm{m}$. The frequency of the electric field $f=1 \mathrm{KHz}$, the number of brushes $N=4$, room temperature $\left(T=26^{\circ} \mathrm{C}\right)$. The error bars are estimated from variation in velocities of several repeat experiments.

The velocities of particles and defects are not only dependent on the thickness of cells but also on the particle size. To further understand their influences on the annihilation dynamics, we also changed both the particle sizes and cell gaps simultaneously at constant aspect ratio $d / R \sim 2$. Fig. 7 shows the dependences of velocities of defects and particles for diameters $6 \mu \mathrm{m}$ (blue circles), $10 \mu \mathrm{m}$ (black squares), and $15 \mu \mathrm{m}$ (red trangles) on the amplitudes of electric fields in cells at cell gaps of $11 \mu \mathrm{m}, 21 \mu \mathrm{m}$ and $32 \mu \mathrm{m}$, respectively. Velocities for sample (A) at $0.3 \mathrm{~V} / \mu \mathrm{m}$ were not recorded, because $E_{\mathrm{c}}$ is in access of this electric field amplitude. It is observed that all the velocities of the negative defects firstly decrease at low electric fields, which is due to the increase of $F_{\mu}$, and subsequently approach saturation for increasing electric field. The 
velocities of the particles are independent on electric field at low voltages and then suddenly decrease to zero. It is worth noting that the velocities of both particles and defects become larger by reducing the particle size as well as the cell gap. This can be attributed to the larger $F_{\text {a }}$ between the particle and the defect core induced by the smaller $D_{i n}$ for smaller particles and thinner cells.

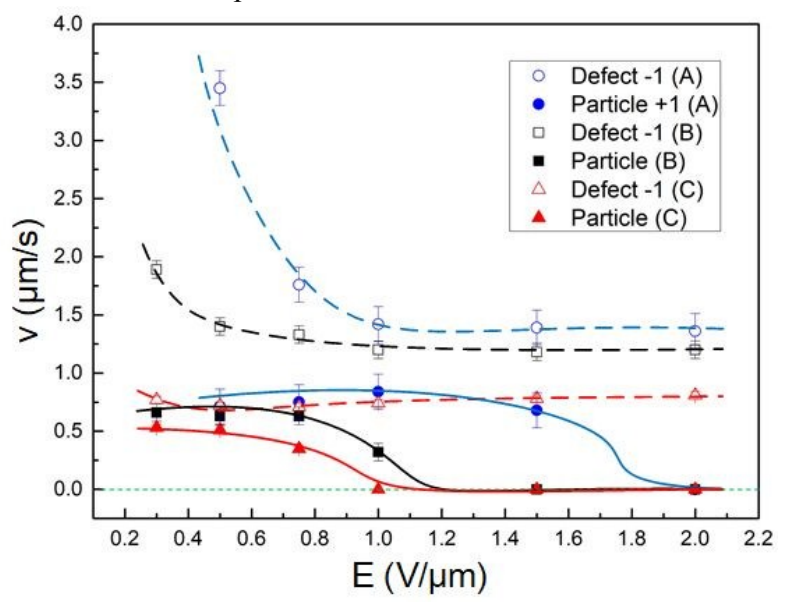

Fig. 7. The dependence of the velocities of negative defects (hollow symbols) and particles (solid symbols) on the amplitude of electric field. (A) $R=6 \mu \mathrm{m}, d=11 \mu \mathrm{m}$. (B) $R=10 \mu \mathrm{m}, d=21 \mu \mathrm{m}$. (C) $R=15$ $\mu \mathrm{m}, d=32 \mu \mathrm{m}$. The frequency of the electric field $f=1 \mathrm{KHz}$, the number of brushes $N=4$, room temperature $\left(T=26^{\circ} \mathrm{C}\right)$. The error bars are estimated from variation in velocities of several repeat experiments.

Another electric field parameter which was varied was the applied sinusoidal wave frequency $f$, from $300 \mathrm{~Hz}$ to $5 \mathrm{KHz}$ as shown in Fig. 8 (a). Although the approaching particle and defect clearly show different speeds, these are independent on electric field frequency. This result is in accordance with the behaviour reported for the annihilation of two opposite defects without particles ${ }^{11}$. In addition, the dependence of the particle-defect annihilation behaviour on the temperature $T$ was investigated. Temperature variation from $26^{\circ} \mathrm{C}$ to $90{ }^{\circ} \mathrm{C}$ will primarily affect the viscosity of the LC material. Since the largest changes of the order parameter occur within the vicinity of a few degrees of the phase transition to the isotropic liquid, the decrease of the order parameter on increasing temperature in the regime investigated will not significantly influence the macroscopic behaviour of the defect annihilation. As depicted in Fig. 8 (b), the velocity at which the annihilating particle and defect approach each other increases nonlinearly with increasing temperature. Such an increase is observed for both particle and defect and can be attributed to an Arrhenius-like decrease of the liquid crystal viscosity ${ }^{11}$.
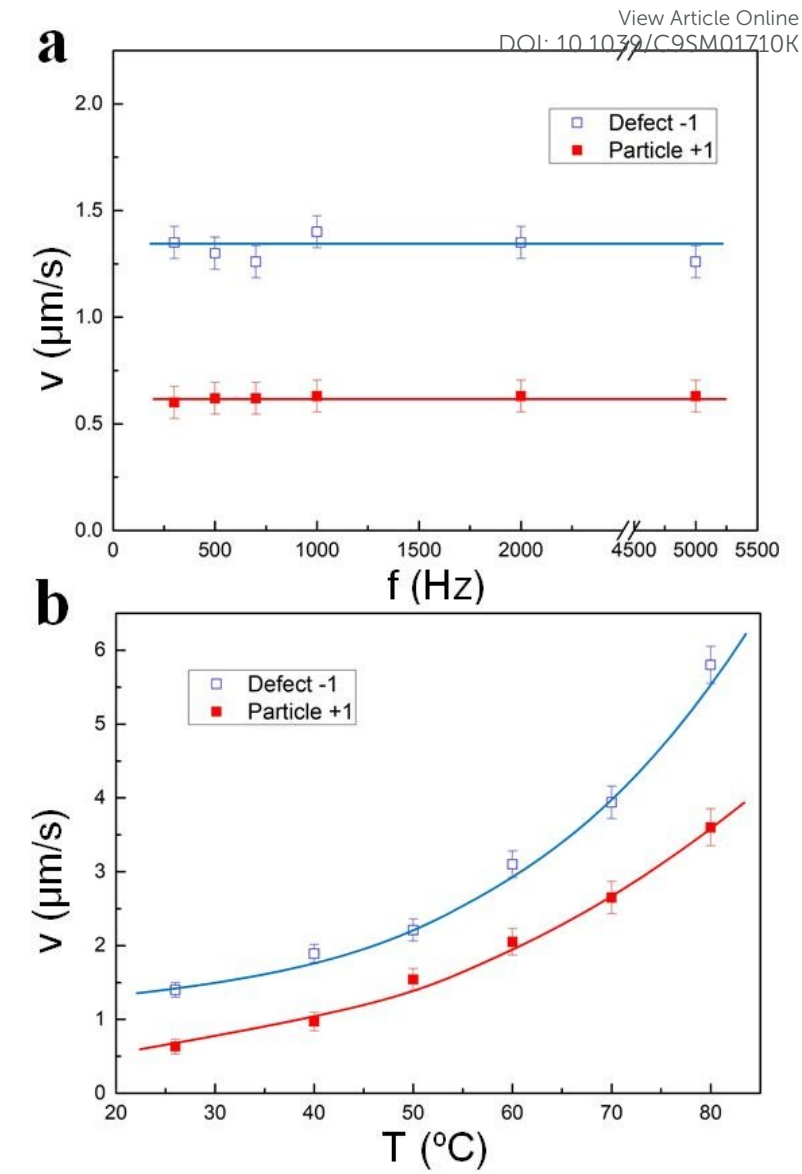

Fig. 8. (a) Frequency dependency of velocities of negative defects (blue squares) and particles (red squares), $T=26{ }^{\circ} \mathrm{C}$. (b) Temperature dependency of velocities of negative defects (blue squares) and particles (red squares), $f=1 \mathrm{KHz}$. The amplitude of the electric field $E=0.5 \mathrm{~V} / \mu \mathrm{m}$, the number of brushes $N=4$, particle diameter $R=10 \mu \mathrm{m}$, cell gap $d=21 \mu \mathrm{m}$. The error bars are estimated from variation in velocities of several repeat experiments.

Finally, the influence of black brushes on the particle-defect annihilation behaviour was investigated. The $s= \pm 1$ defects are always connected via four black brushes when observed through POM, due to the structure of the defect's director field. However, due to the influence of other defects, one defect may not always connect with its counterpart of opposite strength through all four black brushes, as the insets in Fig. 9 (a) show. In our experiment, we found that the number of the black brushes connected with particle $(s=+1)$ and defect $(s=-1)$ would profoundly influence their annihilation velocities. As shown in Fig. 9 (a), (b), and (c), the velocities of both defect and particle decrease with decreasing the number of black brushes connecting them directly together. The smaller velocities of the particle-defect couples which are connected through 2 or 3 black brushes should be understood as the result induced by the influence of other defects nearby which are connected with them through the remaining black brushes as Fig. 9 (d) shows. 

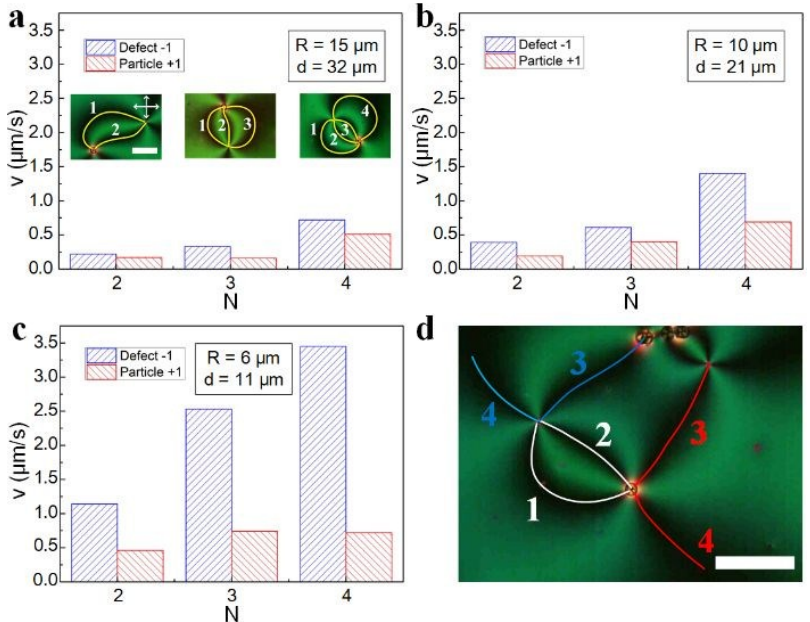

Fig. 9. The velocities of particles (red) and defect (blue) as a function of the number of black brushes, $N$. (a) $R=15 \mu \mathrm{m}, d=32 \mu \mathrm{m}$. The insets in (a) show the couples of particle and defect connected with each other through 2, 3, and 4 black brushes, respectively. The scale bar is $50 \mu \mathrm{m}$. (b) $R=10 \mu \mathrm{m}, d=21 \mu \mathrm{m}$. (c) $R=6 \mu \mathrm{m}, d=11 \mu \mathrm{m}$. (d) A POM image shows a couple of particle and defect connected with each other through two black brushes and with other two black brushes connected with other particles and defects, $R=15 \mu \mathrm{m}, d=$ $32 \mu \mathrm{m}$, scale bar $100 \mu \mathrm{m}$. From (a) to (d), including insets, the electric field $E=0.5 \mathrm{~V} / \mu \mathrm{m}, f=1 \mathrm{KHz}$, temperature $T=26^{\circ} \mathrm{C}$.

\section{Conclusions}

Microparticles with homeotropic surface anchoring dispersed in a nematic LC with a negative dielectric anisotropy act as $s=$ +1 defects and can produce a corresponding $s=-1$ defect nearby by applying an electric field. The annihilation dynamics of couples of $s= \pm 1$ topological defects with and without microparticles were investigated and compared. As previous investigations reported 11,14, 15, 20, positive defects without particles move faster than the negative ones and exhibit a velocity difference which increases by increasing electric fields due to backflow effects. On the contrary, the velocity of positive defects induced by particles is greatly decreased and much slower than that of the negative defects due to the opposed viscous drag force. The velocity of particles is practically independent on the electric field, while the velocity of their corresponding negative defects exhibits a dependency due to the field-dependent size of the defect cores and backflow effects. The annihilation exponents, $\alpha$, of both systems, with and without particles, are equal to 0.5 within experimental error, thus further demonstrating the universality of the proposed scaling behaviour. Furthermore, the effects of particle size, confining cell gap, electric field frequency, temperature, and number of directly connecting brushes on the annihilation dynamics were systematically investigated.

\section{Conflicts of interest}

There are no conflicts to declare.

View Article Online DOI: 10.1039/C9SM01710K

\section{Acknowledgements}

Y. S. gratefully acknowledges the China Scholarship Council (CSC) for kind support.

\section{Notes and references}

1. N. Turok, Physical Review Letters, 1989, 63, 2625.

2. D. Osborne, Proceedings of the Physical Society. Section A, 1950, 63, 909.

3. I. Chuang, R. Durrer, N. Turok and B. Yurke, Science, 1991, 251, 1336-1342.

4. G. Blatter, M. V. Feigel'man, V. B. Geshkenbein, A. I. Larkin and V. M. Vinokur, Reviews of Modern Physics, 1994, 66, 1125.

5. M. Kleman and O. D. Lavrentovich, Philosophical Magazine, 2006, 86, 4117-4137.

6. I. Dierking, Textures of liquid crystals, John Wiley \& Sons, 2003.

7. P.-G. De Gennes and J. Prost, The physics of liquid crystals, Oxford university press, 1995.

8. W. H. Zurek, Nature, 1985, 317, 505-508.

9. N. Fowler and D. I. Dierking, ChemPhysChem, 2017, 18, 812-816.

10. A. Rapini, Journal de Physique, 1973, 34, 629-633.

11. I. Dierking, M. Ravnik, E. Lark, J. Healey, G. Alexander and J. Yeomans, Physical Review E, 2012, 85, 021703.

12. I. Dierking, O. Marshall, J. Wright and N. Bulleid, Physical Review E, 2005, 71, 061709.

13. D. Svenšek and S. Žumer, Physical Review E, 2002, 66, 021712.

14. G. Tóth, C. Denniston and J. M. Yeomans, Physical Review Letters, 2002, 88, 105504.

15. G. Tóth, C. Denniston and J. M. Yeomans, Physical Review E, 2003, 67, 051705.

16. D. Svenšek and S. Žumer, Physical Review Letters, 2003, 90, 155501.

17. A. Bogi, P. Martinot-Lagarde, I. Dozov and M. Nobili, Physical Review Letters, 2002, 89, 225501.

18. P. E. Cladis and H. R. Brand, Physica A: Statistical Mechanics and its Applications, 2003, 326, 322-332.

19. C. Blanc, D. Svenšek, S. Žumer and M. Nobili, Physical Review Letters, 2005, 95, 097802.

20. P. Oswald and J. Ignés-Mullol, Physical review letters, 2005, 95, 027801.

21. T. Yanagimachi, S. Yasuzuka, Y. Yamamura and K. Saito, Journal of the Physical Society of Japan, 2012, 81, 034601.

22. M. Nikkhou, M. Škarabot, S. Čopar, M. Ravnik, S. Žumer and I. Muševič, Nature Physics, 2014, 11, 183.

23. M. Nikkhou, M. Škarabot and I. Muševič, Physical Review E, 2016, 93, 062703.

24. C. Williams, P. Pierański and P. E. Cladis, Physical Review Letters, 1972, 29, 90-92.

25. A. Pargellis, N. Turok and B. Yurke, Physical Review Letters, 1991, 67, 1570-1573. 
26. T. Nagaya, H. Hotta, H. Orihara and Y. Ishibashi, Journal of the Physical Society of Japan, 1991, 60, 1572-1578.

27. T. Nagaya, H. Hotta and H. Oriharaand Yoshihiro Ishibashi, Journal of the Physical Society of Japan, 1992, 61, 35113517.

28. O. Lavrentovich and S. Rozhkov, JETP Lett, 1988, 47.

29. H. Orihara and Y. Ishibashi, Journal of the Physical Society of Japan, 1986, 55, 2151-2156.

30. I. Chuang, N. Turok and B. Yurke, Physical Review Letters, 1991, 66, 2472-2475.

31. I. Chuang, B. Yurke, A. N. Pargellis and N. Turok, Physical Review E, 1993, 47, 3343-3356.

32. D. Pires, J.-B. Fleury and Y. Galerne, Physical Review Letters, 2007, 98, 247801.

33. J. Oh and I. Dierking, Journal of Molecular Liquids, 2018, 267, 315-321.

34. M. A. Gharbi, M. Nobili and C. Blanc, Journal of Colloid and Interface Science, 2014, 417, 250-255.

35. Y. Shen and I. Dierking, Applied Sciences, 2019, 9

36. E. M. Terentjev, Physical Review E, 1995, 51, 1330-1337.

37. O. V. Kuksenok, R. W. Ruhwandl, S. V. Shiyanovskii and E. M. Terentjev, Physical Review E, 1996, 54, 5198-5203.

38. P. Poulin, H. Stark, T. C. Lubensky and D. A. Weitz, Science, 1997, 275, 1770.

39. H. Stark, The European Physical Journal B - Condensed Matter and Complex Systems, 1999, 10, 311-321.

40. H. Stark, Physics Reports, 2001, 351, 387-474.

41. I. I. Smalyukh, A. N. Kuzmin, A. V. Kachynski, P. N. Prasad and O. D. Lavrentovich, Applied Physics Letters, 2005, 86, 021913.

42. T. Araki and H. Tanaka, Physical Review Letters, 2006, 97, 127801.

43. B. Senyuk, Q. Liu, S. He, R. D. Kamien, R. B. Kusner, T. C. Lubensky and I. I. Smalyukh, Nature, 2012, 493, 200.

44. H. Yoshida, K. Asakura, J. Fukuda and M. Ozaki, Nature Communications, 2015, 6, 7180.

45. R. P. Trivedi, I. I. Klevets, B. Senyuk, T. Lee and I. I. Smalyukh, Proceedings of the National Academy of Sciences, 2012, 109, 4744.

46. S. V. Burylov, JETP Letters, 2007, 86, 526-531.

47. S. Grollau, N. L. Abbott and J. J. de Pablo, Physical Review E, 2003, 67, 051703.

48. I. I. Smalyukh, B. I. Senyuk, S. V. Shiyanovskii, O. D. Lavrentovich, A. N. Kuzmin, A. V. Kachynski and P. N. Prasad, Molecular Crystals and Liquid Crystals, 2006, 450, 79/[279]-295/[295].

49. O. D. Lavrentovich, I. Lazo and O. P. Pishnyak, Nature, 2010, 467, 947.

50. O. D. Lavrentovich, Soft Matter, 2014, 10, 1264-1283.

51. J. Oh, H. F. Gleeson and I. Dierking, Physical Review E, 2017, 95, 022703 .

52. X. Tang and J. V. Selinger, Soft Matter, 2017, 13, 54815490.

53. A. J. Vromans and L. Giomi, Soft Matter, 2016, 12, 64906495.

54. X. Tang and J. V. Selinger, Soft Matter, 2019, 15, 587-601.

55. C. Denniston, Physical Review B, 1996, 54, 6272-6275.

56. G. G. Peroli and E. G. Virga, Physical Review E, 1996, 54, 5235-5241.

57. A. N. Pargellis, P. Finn, J. W. Goodby, P. Panizza, B. Yurke and P. E. Cladis, Physical Review A, 1992, 46, 7765-7776.
58. K. Minoura, Y. Kimura, K. Ito, R. Hayakawa and T. Miura, Physical Review E, 1998, 58, 643-649: 10.1039/C9SM01710K

59. H. Pleiner, Physical Review A, 1988, 37, 3986-3992.

60. C. D. Muzny and N. A. Clark, Physical Review Letters, 1992, 68, 804-807.

61. K. Xiao, X. Chen and C.-X. Wu, arXiv preprint arXiv:1907.01347, 2019.

62. T. Yanagimachi, S. Yasuzuka, Y. Yamamura and K. Saito, Journal of the Physical Society of Japan, 2012, 81, 074603. 


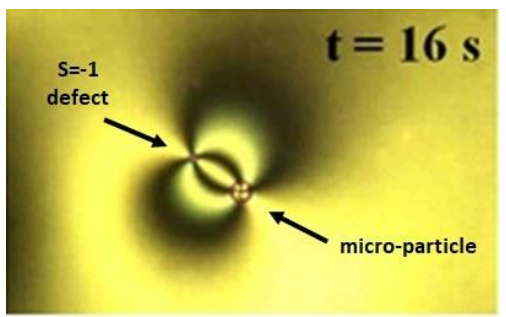

The annihilation dynamics of liquid crystal topological defects with micro-particles is governed by a complex interplay between elastic attraction, backflow, viscous drag forces, confinement and applied electric field conditions. 